PROCEEDINGS OF THE

AMERICAN MATHEMATICAL SOCIETY

Volume 135, Number 3, March 2007, Pages 911-919

S 0002-9939(06)08522-4

Article electronically published on September 11, 2006

\title{
WALDSPURGER'S INVOLUTION AND LIFTING OF CHARACTERS
}

\author{
STEPHEN DEVLIN AND JASON SCHULTZ
}

(Communicated by Dan M. Barbasch)

\begin{abstract}
We show that Adams's $L$-packet of discrete series representations of the two-fold metaplectic cover of $S L_{2}$ coincides with Waldspurger's local near equivalence class.
\end{abstract}

\section{INTRODUCTION}

A fundamental result in the theory of automorphic forms is the notion of strong multiplicity one. Let $F$ be a number field with associated adeles $\mathbb{A}$. Strong multiplicity one states that if $\pi$ and $\pi^{\prime}$ are two automorphic, cuspidal representations of $\mathrm{GL}_{n}(\mathbb{A})$ which agree almost everywhere, then they are the same. That is, if $V$ and $V^{\prime}$ denote, respectively, the space of automorphic cusp forms on which the representations $\pi$ and $\pi^{\prime}$ act, then $\pi \simeq \pi^{\prime}$ almost everywhere implies $V=V^{\prime}$. This result fails to hold when $\mathrm{GL}_{n}(\mathbb{A})$ is replaced with $\mathrm{SL}_{n}(\mathbb{A})([\mathrm{LL}, \S \S 6,7)$.

Consider the metaplectic cover $\widetilde{G L}_{2}\left(\right.$ resp. $\left.\widetilde{S L_{2}}\right)$ of $G L_{2}\left(\operatorname{resp} . S L_{2}\right)$. This is a non-linear, two fold topological covering group, which is also a central extension of $G L_{2}$ (resp. $\left.S L_{2}\right)$ by the group $\{ \pm 1\}$. Strong multiplicity one holds for $\widetilde{G L}_{2}(\mathbb{A})$, and can even be recovered for $\widetilde{S L}_{2}(\mathbb{A})$ with the additional requirement that the central characters of the representations in question be the same (see GPS1). Removing this condition on central characters leads one to 'near equivalence' and the Waldspurger involution ( $\mathrm{W} 2, \S \mathrm{VI})$.

Let SC denote the generalized Shimura correspondence. This correspondence takes an irreducible representation $\pi$ of $G L_{2}$ to an irreducible, genuine representation $\mathrm{SC}(\pi)$ of $\widetilde{G L}_{2}$, or to zero. The local and global Shimura correspondences have been described using character theory and the trace formula (in $[\mathbf{F}$ ), and using L-functions and $\varepsilon$-factors (in GPS3]). Here we are concerned primarily with the description based on character theory. Let $\Theta(\psi)$ denote the Waldspurger correspondence of [W1] and [W2], mapping irreducible representations of $P G L_{2}$ to genuine

Received by the editors February 14, 2005 and, in revised form, October 3, 2005.

2000 Mathematics Subject Classification. Primary 22E50; Secondary 11F70.

This article was co-authored by Jason Schultz in his private capacity. No official support or endorsement by the Social Security Administration or the United States is intended or should be inferred.

(C)2006 American Mathematical Society Reverts to public domain 28 years from publication 
representations of $\widetilde{S L}_{2}$ possessing a $\psi$-Whittaker model. Gelbart and PiatetskiShapiro noted and exploited, in GPS1, the close relationship between these correspondences (see (10) and the comments following). We, in turn, use their results in the sequel.

Given a genuine automorphic cuspidal representation $\tilde{\sigma}$ of $\widetilde{S L}_{2}(\mathbb{A})$, define the near equivalence class of $\tilde{\sigma}, \mathrm{NE}(\tilde{\sigma})$, to be the set of all genuine representations of $\widetilde{S L}_{2}(\mathbb{A})$ which are almost everywhere equivalent to $\tilde{\sigma}$. The extent to which strong multiplicity one fails for $\widetilde{S L}_{2}(\mathbb{A})$ is measured by the size of such near equivalence sets $\operatorname{NE}(\tilde{\sigma})$.

Now let $\tilde{\sigma}$ and $\tilde{\sigma}^{\prime}$ be near equivalent irreducible genuine cuspidal representations of $\widetilde{S L}_{2}(\mathbb{A})$. Fix a character $\psi$ of $\mathbb{A} / F$. There exists a non-square $\xi \in F^{\times}$such that $\tilde{\sigma}$ possesses a $\psi^{\xi}$-Whittaker model. Waldspurger $([\mathrm{W} 2]$, §VI) defines a representation $\pi=S_{\psi}(\tilde{\sigma})$ of $P G L_{2}(\mathbb{A})$ by

$$
S_{\psi}(\tilde{\sigma})=\Theta\left(\psi^{\xi}\right)^{-1}(\tilde{\sigma}) \otimes \chi_{\xi},
$$

where $\chi_{\xi}$ is a certain grossencharacter ([GPS1]). Waldspurger then proves that $\tilde{\sigma}^{\prime}=\Theta\left(\psi^{\xi}\right)\left(\pi \otimes \chi_{\xi}\right)$. From this it follows that each cuspidal automorphic near equivalence class is of the form

$$
\mathrm{NE}(\tilde{\sigma})=\left\{\Theta\left(\psi^{\xi}\right)\left(\pi \otimes \chi_{\xi}\right): \xi \in F^{\times}\right\}
$$

for an automorphic cuspidal representation $\pi$ of $P G L_{2}(\mathbb{A})$. Moreover, this set is finite provided $\tilde{\sigma}$ is not a basic Weil representation of the form $r_{\chi}$, i.e., distinguished in the sense of GPS2].

This suggests a local analogue of near equivalence. Let $F$ be a non-archimedean local field and let $\psi$ be an additive character of $F$. Let $\tilde{\sigma}$ be a genuine, irreducible, admissible representation of $\widetilde{S L}_{2}(F)$ corresponding to the representation $\pi$ of $P G L_{2}(F)$ by SC. Then $\tilde{\sigma}^{\prime}$ is near equivalent to $\tilde{\sigma}$ provided that $\tilde{\sigma}^{\prime}=\Theta\left(\psi^{\xi}\right)\left(\pi \otimes \chi_{\xi}\right)$ for some $\xi \in F^{\times}$(see [W2], $\left.\S \mathrm{VI}\right)$. Here $\chi_{\xi}$ is the quadratic character associated to the extension $F(\sqrt{\xi}) / F$.

Waldspurger proves that if $\tilde{\sigma}$ is a genuine discrete series representation of $S L_{2}(F)$ which is not an odd Weil representation, and which admits a $\psi$-Whittaker model, then there is a non-trivial representative $\xi \in F^{\times}$, modulo squares, for which

$$
\mathrm{NE}(\tilde{\sigma})=\left\{\tilde{\sigma}=\Theta(\psi)(\pi), \quad \tilde{\sigma}^{\prime}=\Theta\left(\psi^{\xi}\right)\left(\pi \otimes \chi_{\xi}\right)\right\} .
$$

The local and global pictures are then connected as follows. Suppose that $\tilde{\sigma}$ is a genuine automorphic cuspidal representation of $\widetilde{S L}_{2}(\mathbb{A})$ that is not a basic Weil representation of the form $r_{\chi}$. Let $\Sigma$ be the finite set of places of $F$ where $\tilde{\sigma}_{\nu}$ is a discrete series representation. Then for each subset $\mathrm{T}$ of $\Sigma$ of even cardinality, define $\tilde{\sigma}^{\mathrm{T}}=\bigotimes \tilde{\sigma}_{\nu}^{\mathrm{T}}$ by

$$
\tilde{\sigma}_{\nu}^{\mathrm{T}}=\left\{\begin{array}{rll}
\Theta\left(\psi_{\nu}^{\xi_{\nu}}\right)\left(\pi_{\nu} \otimes \chi_{\xi_{\nu}}\right) & \text { if } \quad \nu \in \mathrm{T}, \\
\tilde{\sigma}_{\nu} & \text { if } \quad \nu \notin \mathrm{T} .
\end{array}\right.
$$

Then $\mathrm{T} \longrightarrow \tilde{\sigma}^{\mathrm{T}}$ is a bijection between the even subsets of $\Sigma$ and the elements of $\operatorname{NE}(\tilde{\sigma})$.

Recent work of Adams $([\mathrm{A}]$ ), based on character theory and the generalized Shimura correspondence ([KP2], FK]), has led to a natural candidate for an ' $L$ packet' of representations of $\widetilde{S L}{ }_{n}(F)$, the $n$-fold metaplectic cover of $\widetilde{S L}_{n}(F)$.

In this paper, we show that for $n=2$, Adams's $L$-packet coincides with Waldspurger's local near equivalence class (when defined). Since Adams's $L$-packet is 
well defined for $n>2$, it is natural to ask if, analogous to the rank 2 case, the members of the local packets give rise to global near equivalent metaplectic forms.

Start with a representation $\pi$ of $G L_{2}(F)$ with central character $\chi_{\pi}$. Let $\nu$ be a character of $F^{\times}$satisfying $\chi_{\pi}(x \mathrm{I})=\nu\left(x^{2}\right)$. Flicker defines a representation $\tilde{\pi}=$ $L(\pi)$ of $\widetilde{G L}_{2}(F)$ corresponding to $\pi$. Since the central character of $\pi \nu^{-1}$ is trivial, the representation factors to $\overline{\pi \nu^{-1}}$ on $P G L_{2}(F)$. Furthermore, the restriction of $\tilde{\pi}$ from $\widetilde{G L}_{2}(F)$ to $\widetilde{S L}_{2}(F)$ is easy to describe, and the irreducible constituents can be parameterized by characters of $F^{\times}$with fixed restriction to $F^{\times 2}$ (see Corollary 3.2). We choose the constituent corresponding in a natural way to the character $\nu$, and call it $L(\pi, \nu)$. This is a correspondence between genuine representations of $\widetilde{S L}_{2}(F)$ and $P G L_{2}(F)$. Let $\beta$ be a character of $F^{\times}$with $\beta(-1)=-1$. In the same way, $\pi \beta(\nu \beta)^{-1}$ factors to $\overline{\pi \beta(\nu \beta)^{-1}}=\overline{\pi \nu^{-1}}$ on $P G L_{2}(F)$, and corresponds to the genuine representation $L(\pi \beta, \nu \beta)$ on $\widetilde{S L}_{2}(F)$. Hence, there is a natural association between a particular class of genuine representations of $\widetilde{S L}_{2}(F)$ and the representation $\overline{\pi \nu^{-1}}$ of $P G L_{2}(F)$. We show that in the case where $\pi$ is a discrete series representation, the two representations of $\widetilde{S L}_{2}(F)$ corresponding to $\overline{\pi \nu^{-1}}$ are precisely the elements of the local near equivalence class of Waldspurger ([W2, VI). That is, in the above notation, $L(\pi, \nu)=\Theta(\psi)\left(\pi \nu^{-1}\right)$ and $L(\pi \beta, \nu \beta)=$ $\Theta\left(\psi^{\xi}\right)\left(\pi \nu^{-1} \otimes \chi_{\xi}\right)$.

Finally, we note that in recent work, Manderscheid has given another description of the local near equivalence class in the case that both representations are supercuspidal. His description is based on types, the natural inducing data of supercuspidal representations $(\underline{\mathrm{M}}])$.

\section{Preliminaries}

We take $F$ to be a non-archimedean local field with square roots of unity $\mu_{2}=\{ \pm 1\}$. Let $G=G L_{2}(F)$ and $S=S L_{2}(F)$. We consider $\widetilde{G}$ and $\widetilde{S}$, the 2fold metaplectic covers of $G$ and $S$. These topological covering groups are central extensions of $G$ and $S$, respectively, that fit into the exact sequences

$$
\begin{gathered}
1 \longrightarrow \mu_{2} \stackrel{\iota}{\longrightarrow} \widetilde{G} \stackrel{p}{\longrightarrow} G \longrightarrow 1, \\
1 \longrightarrow \mu_{2} \stackrel{\iota}{\longrightarrow} \widetilde{p} \stackrel{p}{\longrightarrow} S \longrightarrow 1 .
\end{gathered}
$$

For a detailed account of the construction of these groups, see [G] and [KP1] (where we take $r=2$ and $c=0$ in their notation). For our purposes it suffices to say that $G$ is determined by a 2-cocycle $c(\cdot, \cdot): G \times G \longrightarrow F^{\times}$. We think of $G$ as the set of pairs $(g, \zeta)$ with $g \in G, \zeta \in \mu_{n}$ and with multiplication given by $(g, \zeta)\left(g^{\prime}, \zeta^{\prime}\right)=$ $\left(g g^{\prime}, \zeta \zeta^{\prime} c\left(g, g^{\prime}\right)\right)$. Then $\widetilde{S}$ is the subset of $\widetilde{G}$ whose elements have a determinant (after composition with $p$ ) equal to 1 . If $H \subset G$, then $\widetilde{H}$ will denote $p^{-1}(H)$, the preimage of $H$ in $\widetilde{G}$. We often write $\zeta$ as an abbreviation for the element $(1, \zeta) \in \widetilde{G}$, where $\zeta \in\{ \pm 1\}$.

Let $F^{\times}$denote the invertible elements in $F$, and $F^{\times 2}$ the set of squares of the elements in $F$. We further denote by $(\cdot, \cdot)$ the Hilbert symbol relative to $F$. This is a non-degenerate pairing, $F^{\times} \times F^{\times} \rightarrow \mu_{2}$, and $F^{\times 2}=\left\{y \in F^{\times}:(y, x)=1 \forall x \in\right.$ $\left.F^{\times}\right\}$. 
Lemma 2.1 ([KP1], $\S 0)$. Let $g, h \in G$, and take $\tilde{g}$ and $\tilde{h}$ to be any inverse images of $g$ and $h$, respectively, in $\widetilde{G}$. Then the $\widetilde{G}$-commutator,

$$
\{g, h\}=\tilde{g} \tilde{h} \tilde{g}^{-1} \tilde{h}^{-1},
$$

is well defined. If $g$ and $h$ commute, then $\{g, h\} \in \iota\left(\mu_{2}\right)$. Furthermore, if $x \in F^{\times}$, then

$$
\{x I, h\}=(\operatorname{det}(h), x) .
$$

The first two parts of following lemma follow from the properties of the Hilbert symbol while the third part is trivial.

Lemma 2.2. Let $Z=\left\{x I: x \in F^{\times}\right\}$be the center of $G, Z^{2}=\left\{y I: y \in F^{\times 2}\right\}$, and $G_{+}=\left\{g \in G: \operatorname{det}(g) \in F^{\times 2}\right\}$. Then

i. The center of $\widetilde{G}$ is $\widetilde{Z}^{2}$.

ii. The center of $\widetilde{G}_{+}$is $\widetilde{Z}$.

iii. $G_{+}=Z \cdot S$.

Finally, we note the exact sequences

$$
\begin{aligned}
& 1 \longrightarrow \mu_{2} \longrightarrow F^{\times} \longrightarrow F^{\times 2} \longrightarrow 1, \\
& 1 \stackrel{\iota}{\longrightarrow} F^{\times 2} \longrightarrow F^{\times} \longrightarrow F^{\times} / F^{\times 2} \longrightarrow 1
\end{aligned}
$$

and their Pontriagin duals

$$
\begin{aligned}
& 1 \longrightarrow \widehat{F^{\times 2}} \longrightarrow \widehat{F^{\times}} \stackrel{\text { res }}{\longrightarrow} \widehat{\mu_{2}} \longrightarrow 1, \\
& 1 \longrightarrow \widehat{F^{\times} / F^{\times 2}} \longrightarrow \widehat{F^{\times}} \stackrel{\text { res }}{\longrightarrow} \widehat{F^{\times 2}} \longrightarrow 1 .
\end{aligned}
$$

We therefore identify the characters of $F^{\times}$that are trivial on $\mu_{2}$ with the squares of characters of $F^{\times}$. That is,

Lemma 2.3. Let $\lambda \in \widehat{F^{\times}}$. Then $\lambda=\nu^{2}$ for some $\nu \in \widehat{F^{\times}}$if and only if $\lambda(-1)=1$.

\section{REPRESENTATION THEORY}

Let $\tilde{\pi}$ be a representation of a subgroup of $\widetilde{G}$ with central character $\chi_{\tilde{\pi}}$. We say that $\tilde{\pi}$ (resp. $\chi_{\tilde{\pi}}$ ) is genuine provided it acts via the non-trivial character $\varepsilon$ of $\mu_{2}$.

Suppose that $\chi$ is a genuine character of $\widetilde{Z}^{2}$ (the center of $\widetilde{G}$ ) and $\mu$ is a character of $\widetilde{Z}$ (the center of $\widetilde{G}_{+}$) which restricts to $\chi$ on $\widetilde{Z}^{2}$. Let $\tilde{g}$ be an element of $\widetilde{G}$ with $\operatorname{det}(\tilde{g})=a$. Then for $\tilde{z} \in \widetilde{Z}$, define a character ${ }^{\tilde{g}} \mu$ of $\widetilde{Z}$ by ${ }^{\tilde{g}} \mu(\tilde{z})=\mu\left(\tilde{g}^{-1} \tilde{z} \tilde{g}\right)$. Taking $\tilde{z}=(x I, \zeta)$ for some $x \in F^{\times}$, and since $\mu$ is genuine, (2) gives

$$
\tilde{g} \mu(\tilde{z})=\mu\left(\tilde{g}^{-1} \tilde{z} \tilde{g}\right)=\mu(\tilde{z}(a, x))=\mu(\tilde{z}) \varepsilon((a, x)) .
$$

Given $a \in F^{\times}$, we let ${ }^{a} \mu=\tilde{g}^{\tilde{g}} \mu$, where $\tilde{g}$ is any element of $\widetilde{G}$ with $\operatorname{det}(\tilde{g})=a$. By the properties of the Hilbert symbol, $\mu \neq \tilde{g}_{\mu}$ provided $a \notin F^{\times 2}$. Moreover, the conjugates of $\mu$ by a set $X$ of coset representatives of $\widetilde{G} / \widetilde{G}_{+}$exhaust the characters of $\widetilde{Z}$ that restrict to $\chi$ on $\widetilde{Z}^{2}$. The determinants of the elements in $X$ in turn determine a set $Y$ of coset representatives of $F^{\times} / F^{\times 2}$. For $\chi \in \widehat{\widetilde{Z}}^{2}$, we let $\Omega_{\chi}=$ $\left\{\nu \in \widehat{\widetilde{Z}}:\left.\nu\right|_{\widetilde{Z}^{2}}=\chi\right\}$. If $\mu$ is any element of $\Omega_{\chi}$, we have

$$
\Omega_{\chi}=\left\{{ }^{\tilde{g}} \mu: \tilde{g} \in X\right\}=\left\{{ }^{a} \mu: a \in Y\right\} .
$$

Finally, let $\tilde{\pi}$ be an irreducible representation of $\widetilde{G}$ with central character $\chi_{\tilde{\pi}}$. We can use Clifford theory to describe the restriction of $\tilde{\pi}$ from $\widetilde{G}$ to $\widetilde{S}$. If $\tilde{\sigma}_{+}$is 
an irreducible constituent of $\operatorname{Res} \widetilde{G}_{\widetilde{G}_{+}}^{\widetilde{\pi}} \tilde{\pi}$, then its conjugate representations $\left\{\tilde{g}^{\tilde{\sigma}} \tilde{\sigma}_{+}\right.$: $\tilde{g} \in X\}$ are all distinct, since the central characters of these representations are precisely the elements of $\Omega_{\chi_{\tilde{\pi}}}$. Since no two elements of $\Omega_{\chi_{\tilde{\pi}}}$ are the same, all the conjugates of $\tilde{\sigma}_{+}$are disjoint. Summarizing, we have (cf. (GPS1], p. 101)

\section{Theorem 3.1.}

$$
\operatorname{Res} \widetilde{G}_{+}^{\widetilde{G}} \tilde{\pi}=\bigoplus_{\tilde{g} \in X} \tilde{g}^{\tilde{\sigma}} \tilde{\sigma}_{+} \cdot
$$

Moreover, any of the ${ }^{\tilde{g}} \tilde{\sigma}_{+}$induce irreducibly to $\tilde{\pi}$ on $\widetilde{G}$. Furthermore, if $V_{\tilde{\pi}}$ is the space of $\tilde{\pi}, \nu \in \widehat{\widetilde{Z}}$, and we let $V^{\nu}=\left\{v \in V_{\tilde{\pi}}: \tilde{\pi}(\tilde{z}) v=\nu(\tilde{z}) v \forall \tilde{z} \in \widetilde{Z}\right\}$, then $V^{\nu}$ carries a representation of $\widetilde{G}_{+}$, namely $\left.\tilde{\pi}\right|_{\widetilde{G}_{+}}$. This gives

\section{Corollary 3.2.}

$$
\operatorname{Res} s_{\widetilde{G}_{+}}^{\widetilde{G}} \tilde{\pi}=\bigoplus_{\tilde{g} \in X}{ }^{\tilde{g}} \tilde{\sigma}_{+}=\bigoplus_{\mu \in \Omega_{\chi}}\left(V^{\mu},\left.\tilde{\pi}\right|_{\widetilde{G}_{+}}\right)=\bigoplus_{a \in Y}{ }^{a} \tilde{\sigma}_{+},
$$

with equivalences given by matching central characters.

Since $\widetilde{S}$ and $\widetilde{G}_{+}$differ by the abelian group $\widetilde{Z}$ (Lemma 2.2 ii), there is no further reducibility when $\tilde{\pi}$ is restricted to $\widetilde{S}$. We write

$$
\operatorname{Res} \underset{\widetilde{S}}{\widetilde{\pi}} \tilde{\pi}=\bigoplus_{a \in Y}{ }^{a} \tilde{\sigma}
$$

where $^{a} \tilde{\sigma}={ }^{a} \tilde{\sigma}_{+} \mid \widetilde{S}$

\section{LIFTING}

We recall the theory of lifting of representations between $\widetilde{G}$ and $G$ due to Flicker $\left([\mathrm{F}]\right.$ ). We write $\Theta_{\pi}$ for the global character of a representation $\pi$, considered as a function on the regular semisimple elements of the group.

Given a regular element $g$ with eigenvalues $x_{1} \neq x_{2}$, the Weyl denominator is defined to be $\Delta(g)=\left|\frac{\left(x_{1}-x_{2}\right)^{2}}{x_{1} x_{2}}\right|^{\frac{1}{2}}$. If $g$ is conjugate to $\operatorname{diag}(x, y)$, we let $g^{\prime}=$ $\operatorname{diag}(-x, y)$. Furthermore, let $b=\frac{2}{|2|_{F}}, \zeta_{g} \in\{ \pm 1\}$ as defined in [Sc], 1.2.

Definition 4.1. Suppose $\tilde{\pi}$ is an irreducible admissible representation of $\widetilde{G}$ with central character $\chi_{\tilde{\pi}}$, and $\pi$ is an admissible representation of $G$ with central character $\chi_{\pi}$. Assume that

$$
\chi_{\tilde{\pi}}\left(x^{2}, 1\right)=\chi_{\pi}(x I) \forall x \in F^{\times} .
$$

Then we say that $\tilde{\pi}$ and $\pi$ correspond via Flicker's correspondence provided that whenever $g^{2}$ is regular we have

$$
\Delta\left(g^{2}\right) \Theta_{\tilde{\pi}}\left(g^{2}, \zeta_{g}\right)=\left\{\begin{array}{cl}
b \Delta(g) \Theta_{\pi}(g) & \text { if } g \text { is elliptic, } \\
b\left(\Delta(g) \Theta_{\pi}(g)+\Delta\left(g^{\prime}\right) \Theta_{\pi}\left(g^{\prime}\right)\right) & \text { if } g \text { is hyperbolic. }
\end{array}\right.
$$

If $\pi$ and $\tilde{\pi}$ correspond, we say that $\tilde{\pi}$ is the lift of $\pi$, and we write $\tilde{\pi}=L(\pi)$.

Theorem $4.2(\overline{\mathrm{F}}, 5.2)$. The correspondence given by $\tilde{\pi}=L(\pi)$ is a bijection between irreducible admissible representations $\pi$ of $G$ satisfying $\chi_{\pi}(-I)=1$ and genuine irreducible admissible representations $\tilde{\pi}$ of $\widetilde{G}$. 
Remark 4.3. $L(\pi)$ is a discrete series representation if and only if $\pi$ is, and the correspondence is a bijection between the discrete series of $G$ and the genuine discrete series of $\widetilde{G}$. On the other hand, if $\tilde{\pi}$ is supercuspidal, with $\tilde{\pi}=L(\pi)$, then $\pi$ is supercuspidal, but not conversely.

\section{AdAMS'S CONSTRUCTION}

We recall Adams's notion of a conjectural $L$-packet of representations of $\widetilde{S}$ from A in the special case $n=2$.

Fix an additive character $\psi$ of $F$ and let $\gamma_{F}$ be the Weil index of the map $x \mapsto \psi\left(x^{2}\right)$ (see [We]).

Definition 5.1. Define the gamma factor to be

$$
\gamma_{F}(x, \psi)=\frac{\gamma_{F}\left(\psi^{x}\right)}{\gamma_{F}(\psi)}
$$

where $\psi^{x}(a)=\psi(x a)$.

Definition 5.2. Define a character $\rho_{\gamma}^{\psi}$ of $\widetilde{Z}$, the center of $\widetilde{G}_{+}$, by

$$
\rho_{\gamma}^{\psi}(x I, \zeta)=\gamma_{F}(x, \psi) \varepsilon(\zeta)
$$

The fact that this defines a genuine character follows from the properties of the gamma factor listed in $\left[\mathrm{R}\right.$. We note that the characters of $F^{\times}$are in bijection with the genuine characters of $\widetilde{Z}$ via $\nu \mapsto \chi_{\nu}$, with $\chi_{\nu}$ defined by

$$
\chi_{\nu}(x I, \zeta)=\nu(x) \rho_{\gamma}^{\psi}(x I, \zeta) .
$$

Definition 5.3. Given the representation $L(\pi)$ of $\widetilde{G}$, we let $L_{+}(\pi, \nu)$ denote that irreducible constituent in the restriction of $L(\pi)$ to $\widetilde{G}_{+}$with central character $\chi_{\nu}$. Furthermore, let $L(\pi, \nu)$ be the restriction of $L_{+}(\pi, \nu)$ to $\widetilde{S}$.

Remark 5.4. Comparing our notation to that of GPS1, $\S 2$, we have $L(\pi)=\bar{\pi}$, $L(\pi, \nu)=\operatorname{Res}_{\chi_{\nu}} \bar{\pi}$, and $\nu=\omega$. Here $\operatorname{Res}_{\chi_{\nu}}$ denotes restricting from $\widetilde{G}$ to $\widetilde{G}_{+}$, choosing the constituent with central character $\chi_{\nu}$, and further restricting to $\widetilde{S}$.

Now, suppose a representation $\pi$ has central character $\chi_{\pi}$ with $\chi_{\pi}(-I)=1$. Let $\nu$ be a character of $F^{\times}$such that $\chi_{\pi}(x I)=\nu^{2}(x)$ for all $x \in F^{\times}$(by (3) ), and let $\alpha$ be a character of $F^{\times}$. If we let $\pi \alpha$ denote $\pi \otimes(\alpha \circ$ det $)$, then the central character satisfies $\chi_{\pi \alpha}(-I)=1$, and $\chi_{\pi \alpha}(x I)=(\nu \alpha)^{2}(x)$.

Lift $\pi \alpha$ to an irreducible genuine representation $L(\pi \alpha)$ of $\widetilde{G}$. Considering the central character of $\pi \alpha(\nu \alpha)^{-1}$, we have

$$
\chi_{\pi \alpha(\nu \alpha)^{-1}}(x I)=\chi_{\pi}(x I) \nu^{-1}\left(x^{2}\right)=1,
$$

so $\pi \alpha(\nu \alpha)^{-1}$ factors to $\overline{\pi \nu^{-1}}$ on $P G L_{2}(F)$. Let $\chi_{\nu \alpha}=\nu \alpha \rho_{\gamma}^{\psi}$ as in (6). In a natural way, $L(\pi \alpha, \nu \alpha)$ corresponds to $\overline{\pi \nu^{-1}}$.

Starting with a pair $(\pi, \nu)$ as above, we get a correspondence between irreducible representations of $P G L_{2}(F)$ and irreducible genuine representations of $\widetilde{S}$. Since $L(\pi \alpha, \nu \alpha)=L(\pi, \nu)$ if $\alpha \in \widehat{F^{\times}}$is a square, the correspondence is parameterized by the characters of $\widehat{F^{\times}} / \widehat{F^{\times 2}} \cong \widehat{\mu_{2}}$ (see (3)). If we let $\beta$ be the non-trivial character of $\mu_{2}$, then we have (cf. $\left.\mathrm{A}\right]$ )

$$
\overline{\pi \nu^{-1}} \longleftrightarrow\left\{L(\pi \alpha, \nu \alpha): \alpha \in \widehat{\mu_{2}}\right\}=\{L(\pi, \nu), L(\pi \beta, \nu \beta)\} .
$$


This is the $n=2$ case of the set that Adams refers to in [A] as a conjectural $L$ packet. In $[\mathrm{Sc}$, Schultz used this packet to define a stable character correspondence between $P G L_{2}(F)$ and $\widetilde{S L}_{2}(F)$, which was generalized for $n>2$ in $\mathrm{A}$.

\section{The CORRESPONDENCE}

We recall Waldspurger's notion of near equivalence.

Theorem 6.1 (Waldspurger, W2, IV, Théorème 1). Suppose that $\tilde{\sigma}$ is an irreducible genuine discrete series representation of $\widetilde{S}$ possessing a $\psi$-Whittaker model. Then there exists a representation $\pi$ of $P G L_{2}(F)$ for which $\tilde{\sigma}=\Theta(\psi)(\pi)$. Define the local near equivalence class of Waldspurger to be the set (W2, VI)

$$
N E(\tilde{\sigma})=\left\{\Theta(\psi)(\pi), \Theta\left(\psi^{\xi}\right)\left(\pi \otimes \chi_{\xi}\right)\right\} .
$$

Here $\xi$ is a non-trivial representative of $F^{\times} / F^{\times 2}$ for which $\Theta\left(\psi^{\xi}\right)\left(\pi \otimes \chi_{\xi}\right)$ admits $a \psi^{\xi}$-Whittaker model and $\chi_{\xi}=\gamma(\cdot, \psi) \gamma\left(\cdot, \psi^{\xi}\right)$.

We refer to the elements of $N E(\tilde{\sigma})$ as a representation and its image under the Waldspurger involution.

Let $\tilde{\sigma}$ be a discrete series representation of $\widetilde{S}$ and fix a character $\psi$ such that $\tilde{\sigma}$ admits a $\psi$-Whittaker model. Let $\nu$ be a multiplicative character such that $\chi_{\nu} \tilde{\sigma}$ extends to $\widetilde{G}_{+}$. Then $\chi_{\nu} \tilde{\sigma}$ induces irreducibly to a representation $\tilde{\pi}$ of $\widetilde{G}$, which corresponds via Theorem 4.2 to a representation $\pi$ of $G$. We write $\tilde{\pi}=L(\pi)$ and have $\chi_{\pi}(x I)=\nu\left(x^{2}\right)$, and $\tilde{\sigma}=L(\pi, \nu)$.

Lemma 6.2. $L(\pi, \nu)=\Theta(\psi)\left(\overline{\pi \nu^{-1}}\right)$.

Proof. Note that $\pi \nu^{-1}$ factors to the representation $\overline{\pi \nu^{-1}}$ of $P G L_{2}(F)$ by (77). We have maps

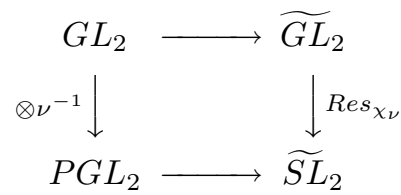

given by

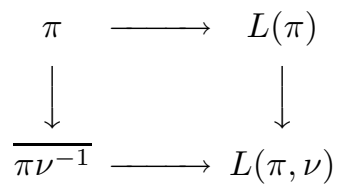

It follows from [GPS1], $\S 2$ (with, comparing their notation to ours, $\mu=\chi_{\nu}, \chi_{\psi}=$ $\gamma(\cdot, \psi)$, and $\omega=\nu)$, that the map $\overline{\pi \nu^{-1}} \mapsto L(\pi, \nu)$ on the bottom of the diagram is the correspondence of Theorem 6.1 and $L(\pi, \nu)=\Theta(\psi)\left(\overline{\pi \nu^{-1}}\right)$.

Now we apply the construction of section 5 . Let $\beta$ be a multiplicative character of $F^{\times}$with $\beta(-1)=-1$ (so $\beta$ determines the non-trivial character of $\mu_{2}$ ). Using the construction in diagram (10) with the character $\nu \beta$ in place of $\nu$, we get a new diagram with $\pi \beta$ and $L(\pi \beta)$ on the top, and $\overline{\pi \beta(\nu \beta)^{-1}}=\overline{\pi \nu^{-1}}$ and $L(\pi \beta, \nu \beta)$ 
$\left(=\operatorname{Res}_{\chi_{\nu \beta}} L(\pi \beta)\right)$ on the bottom:

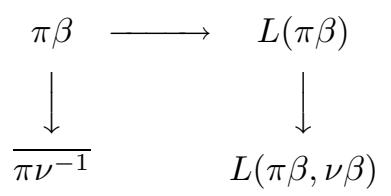

Lemma 6.3. $L(\pi \beta, \nu \beta)$ and $L(\pi, \nu)$ are inequivalent.

Proof. Their central characters, $\chi_{\nu}$ and $\chi_{\nu \beta}$, are different. We have

$$
\chi_{\nu \beta}(-I, \zeta)=\nu \beta(-1) \rho_{\gamma}(-1, \zeta)=-\nu(-1) \rho_{\gamma}(-1, \zeta)=-\chi_{\nu}(-I, \zeta) .
$$

It follows that $L(\pi \beta, \nu \beta)$ does not admit a $\psi$-Whittaker model, since if it did, the above diagram would commute, the bottom arrow would be Waldspurger's map, and we would get $\Theta(\psi)\left(\overline{\pi \nu^{-1}}\right)=L(\pi, \nu)=L(\pi \beta, \nu \beta)$, contradicting the lemma.

The connection between the near equivalence sets of Waldspurger and the correspondence of Definition 5.3 is now apparent.

Theorem 6.4. With notation as above,

$$
N E(\tilde{\sigma})=\{L(\pi, \nu), L(\pi \beta, \nu \beta)\} .
$$

Proof. Lemma 6.2 gives $\Theta(\psi)\left(\overline{\pi \nu^{-1}}\right)=L(\pi, \nu)$, so it remains to show that

$$
\Theta\left(\psi^{\xi}\right)\left(\overline{\pi \nu^{-1}} \otimes \chi_{\xi}\right)=L(\pi \beta, \nu \beta)
$$

To this end, choose a non-trivial representative $\xi$ of $F^{\times} / F^{\times 2}$ such that $L(\pi \beta, \nu \beta)$ admits a $\psi^{\xi}$-Whittaker model. We build a diagram exactly as in Lemma 6.2, but using $\psi^{\xi}$ in place of $\psi$ in Definitions 5.1 and 5.2 , and using $\nu \beta \chi_{\xi}$ in place of $\nu$. We have

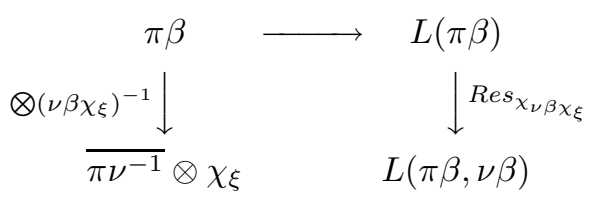

As in Lemma 6.2 we have $\Theta\left(\psi^{\xi}\right)\left(\overline{\pi \nu^{-1}} \otimes \chi_{\xi}\right)=L(\pi \beta, \nu \beta)$. (Note that

$$
\chi_{\nu \beta \chi_{\xi}}(x I, \zeta)=\nu \beta(x) \rho_{\gamma}^{\psi}(x I, \zeta),
$$

which is $\chi_{\nu \beta}$ using $\psi$ in (6).)

Finally, we note that much has been done in the direction of a general theory of lifting of representations (on the level of characters) in [FK], KP1], and [KP2]. Assuming such a lifting theory, the constructions of sections 3 and 5 are well defined for $n>2$. In particular, given a representation $\pi$ of $G L_{n}(F)$ with $\chi_{\pi}$ trivial on the $n$-th roots of unity $\mu_{n}(F)$, a character $\nu$ of $F^{\times}$satisfying $\chi_{\pi}(x I)=\nu\left(x^{n}\right)$, and a lift $L(\pi)$ to $\widetilde{G L}_{n}(F)$, Adams has defined a correspondence between representations of $P G L_{n}(F)$ and $\widetilde{S L}{ }_{n}(F)$ parameterized by the characters of $\mu_{n}(F)$ (see [], $\S 5$, and (8)):

$$
\overline{\pi \nu^{-1}} \longleftrightarrow\left\{L(\pi \alpha, \nu \alpha): \alpha \in \widehat{\mu_{n}(F)}\right\} .
$$


If $\pi$ is a discrete series representation, then the lift $L(\pi)$ is defined, and the set on the right-hand side consists of $n$ distinct non-zero representations. A natural question is whether the elements of this packet lead, by analogy, to global packets and near equivalent metaplectic forms.

\section{REFERENCES}

[A] J. Adams, Characters of Covering Groups of $S L(n)$, J. Inst. Math. Jussieu 2 (2003) no. 1, 1-21. MR1955205 (2004a:22016)

[F] Y. Flicker, Automorphic Forms on Covering Groups of GL(2), Invent. Math. 57 (1980), 119-182. MR 0567194 (81m:10057)

[FK] Y. Flicker and D. Kazhdan, Metaplectic Correspondence, Inst. Hautes Études Sci. Publ. Math. 64 (1986), 53-110. MR0876160 (88d:11049)

[G] S. Gelbart Weil's Representation and the Spectrum of the Metaplectic Group, Springer Lecture Notes in Mathematics, Vol. 530, Springer-Verlag (1976). MR0424695 (54:12654)

[GHPS] S. Gelbart, R. Howe, and I. Piatetski-Shapiro, Uniqueness anand Existence of Whittaker Models for the Metaplectic Group, Israel. Jour. of Math. 34 (1979), nos. 1-2, 21-37. MR0571393 (81j:22021)

[GPS1] S. Gelbart and I. Piatetski-Shapiro, Some remarks on metaplectic cu cusp forms and the correspondences of Shimura and Waldspurger, Israel. Jour. of Math. 44 (1983), no. 2, 97-126. MR 0693355 (84g:10054)

[GPS2] S. Gelbart and I. Piatetski-Shapiro, Distinguished representations and modular forms of half-integral weight, Inv. Math. 59 (1980), 145-188. MR0577359 (82b:10035)

[GPS3] S. Gelbart and I. Piatetski-Shapiro, On Shimura's correspondence for modular forms of half-integral weight, Proc. Colloquium on Automorphic Forms, Representation Theory, and Arithmetic, Bombay, Tata Institute Research Studies in Mathematics 10, SpringerVerlag (1981). MR0633657 (83b:10032)

[KP1] D.A. Kazhdan and S.J. Patterson, Metaplectic Forms, Publ. Math. I.H.E.S. 59 (1984), 35-142. MR0743816 (85g:22033)

[KP2] D.A. Kazhdan and S.J. Patterson, Towards a Generalized Shimura Correspondence, Adv. in Math. 60(2) (1986), 161-234. MR0840303 (87m:22050)

[LL] R.P. Langlands and J.-P. Labesse, L-indistinguishability for SL(2), Canad. J. Math. 31 (1979), 726-785. MR0540902 (81b:22017)

[M] D. Manderscheid, Waldspurger's Involution and Types, J. London Math. Soc. (2) 70 (2004), no. 3, 567-585. MR2096864 (2005j:11037)

[R] R. Ranga Rao, On some explicit formulas in the theory of Weil representations, Pac. J. Math 157 (1993), no. 2, 355-371. MR.1197062 (94a:22037)

[Sc] J. Schultz, Lifting of characters on $\overline{S L(2)}$ and $S O(1,2)$ over a non-archimedean local field, Ph.D. Thesis, University of Maryland (1998).

[W1] J.L. Waldspurger, Correspondence de Shimura, J. Math. Pures et Appl. 59 (1980), 1-133. MR 0577010 (83f:10029)

[W2] J.L. Waldspurger, Correspondence de Shimura et quaternions, Forum Math. 3 (1991), 219-307. MR1103429 (92g:11054)

[We] A. Weil, Sur certaines groupes d'opérateurs unitaires, Acta Math. 11 (1964), 143-211. MR0165033 (29:2324)

Department of Mathematics, University of San Francisco, 2130 Fulton Street, San Francisco, CALifornia 94117

E-mail address: smdevlin@usfca.edu

Office of the Chief Actuary, Social Security Administration, Baltimore, Maryland 21235 